\title{
Relativistic effects and fully spin-polarized Fermi surface at the Tl/Si(111) surface
}

\author{
Julen Ibañez-Azpiroz, ${ }^{1,2}$ Asier Eiguren, ${ }^{1,2}$ and Aitor Bergara ${ }^{1,2,3}$ \\ ${ }^{1}$ Materia Kondentsatuaren Fisika Saila, Zientzia eta Teknologia Fakultatea, Euskal Herriko Unibertsitatea, \\ 644 Postakutxatila, 48080 Bilbao, Basque Country, Spain \\ ${ }^{2}$ Donostia International Physics Center (DIPC), Paseo Manuel de Lardizabal 4, 20018 Donostia/San Sebastian, Spain \\ ${ }^{3}$ Centro de Física de Materiales CFM - Materials Physics Center MPC, Centro Mixto CSIC-UPVIEHU, Edificio Korta, \\ Avenida de Tolosa 72, 20018 Donostia, Basque Country, Spain
}

(Received 18 May 2011; revised manuscript received 18 July 2011; published 19 September 2011)

\begin{abstract}
We present a detailed analysis of the relativistic electronic structure and the momentum-dependent spinpolarization of the $\mathrm{Tl} / \mathrm{Si}(111)$ surface. Our first-principle calculations reveal the existence of fully spin-polarized electron pockets associated to the huge spin-splitting of metallic surface bands. The calculated spin-polarization shows a very complex structure in the reciprocal space, strongly departing from simple theoretical model approximations. Interestingly, the electronic spin-state close to the Fermi surface is polarized along the surface perpendicular direction and reverses its orientation between different electron pockets.

DOI: 10.1103/PhysRevB.84.125435

PACS number(s): 73.20.At, 72.25.-b, 75.76.+j, 73.50.-h
\end{abstract}

\section{INTRODUCTION}

The role played by the electronic spin in nominally nonmagnetic surfaces has attracted a considerable interest in the last two decades due to the potential applications of these systems in the emergent field of spintronics. ${ }^{1-4}$ Soon after the discovery of a spin-splitted surface band in the $\mathrm{Au}(111)$ metallic surface, ${ }^{5}$ a large variety of surfaces has been intensively examined, both theoretically and experimentally. ${ }^{6-10}$ It is now well established that the origin of the surface electron spin-splitting resides on the lack of the inversion symmetry close to the surface area. Interestingly, recent model calculations ${ }^{11,12}$ suggest the important role played by the in-plane inversion asymmetry in the enhancement of the spin-splitting magnitude, as well as in the determination of the spin-polarization structure.

Among the many surfaces exhibiting spin-splitting phenomena, semiconductor substrates covered by a single heavyelement overlayer demonstrate specially encouraging properties for possible spintronic applications. ${ }^{13,14}$ On one hand, these types of surfaces have been recently found to exhibit exceptional relativistic effects, ${ }^{15,16}$ inducing spin-orbit energy shifts two orders of magnitude bigger than those found at normal semiconductor heterojunctions. From the practical point of view, heavy elements such as $\mathrm{Tl}$ or $\mathrm{Sb}$ are widely used in electronic instruments such as infrared detectors ${ }^{17}$ or Halleffect devices. ${ }^{18}$ Another important property of semiconductor surfaces is the band gap associated to the semiconductor substrate, which ensures the two-dimensional character of the transport properties and the absence of any appreciable bulk contribution, both conditions being indispensable for an effective manipulation of the surface spin-state.

$\mathrm{The} \mathrm{Tl} / \mathrm{Si}(111)$ surface is an outstanding example in which the spin-orbit interaction plays a critical role in determining the transport properties of the system. The honeycomb layered structure of the $\mathrm{Si}(111)$ substrate induces a singular spinpattern in momentum space that departs from simple pictures such as the Rashba model. ${ }^{19}$ This peculiar property is farther enhanced by the strong relativistic effects associated to the $\mathrm{Tl}$ overlayer, producing a highly complex spin-configuration in the neighborhood of the Fermi level. In this paper, we investigate the nature of the spin-orbit interaction on two different terminations of the $\mathrm{Tl} / \mathrm{Si}(111)$ surface describing the relativistic electronic structure and the momentum-dependent spin-polarization.

\section{COMPUTATIONAL METHOD}

We have considered the noncollinear DFT formalism and plane waves as basis functions for the expansion of the Kohn-Sham orbitals. ${ }^{20,21}$ The convergence of the plane wave basis has been achieved with an energy cutoff of $50 \mathrm{Ry}$. The integrations over the surface Brillouin zone have been performed using the tetrahedron method ${ }^{22}$ considering a $32 \times 32$ Monkhorst-Pack mesh. ${ }^{23}$ The exchange-correlation energy has been approximated within the PBE-GGA parametrization. ${ }^{24,25}$

We used norm-conserving fully relativistic pseudopotentials as illustrated in Refs. 26 and 27. These pseudopotentials describe the relativistic effects up to order $1 / c^{2}$, including the mass-velocity, the Darwin and the spin-orbit coupling terms. ${ }^{28}$ The electron wave functions are treated within the spinor formalism in order to properly incorporate the noncollinear effects associated to the spin-orbit interaction.

In this paper, we consider two different surface terminations designated as Tl/Si(111)A and Tl/Si(111)B (see Fig. 1). Both terminations were modeled within the repeated slab approach consisting of 40 atom layers, imposing a relaxation criterion of $\left|F_{i}\right|<10^{-4}$ Ry a.u. $^{-1}$. Previous experimental and theoretical investigations $\mathrm{s}^{29,30}$ demonstrated that the $\mathrm{Tl} / \mathrm{Si}(111) \mathrm{A}$ termination is the most stable. However, in our calculations, we find that the $\mathrm{Tl} / \mathrm{Si}(111) \mathrm{B}$ termination is energetically competitive, with a tiny energy cost of $\Delta E_{s} \simeq 10^{-3}$ Ry per Tl atom.

\section{RESULTS}

\section{A. $\mathbf{T l} / \mathrm{Si}(\mathbf{1 1 1}) \mathrm{A}$}

Figure 2 illustrates our results for the electronic structure of the $\mathrm{Tl} / \mathrm{Si}(111) \mathrm{A}$ termination. The scalar relativistic calculation (without spin-orbit term) produces two surface states, labeled as $S_{A 1}$ and $S_{A 2}$ (see Fig. 2). This calculation predicts a semiconductor state with an energy gap of $\sim 0.2 \mathrm{eV}$, since 


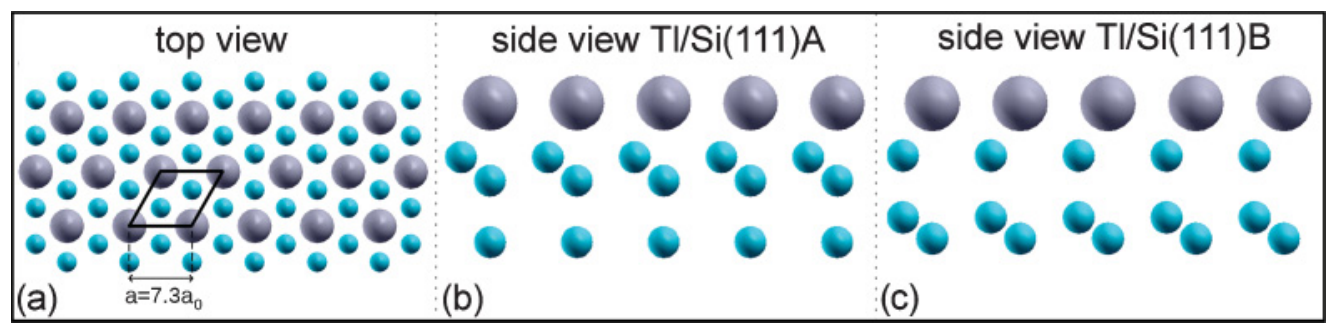

FIG. 1. (Color online) (a) Top view of the Tl/Si(111) surface. Big (gray) spheres represent the Tl surface monolayer, while the small (blue) ones are the Si substrate layers. The solid (black) lines denote the projection of the surface unit cell. (b) and (c) Side view of the two surface terminations considered, Tl/Si(111)A and Tl/Si(111)B.

neither $S_{A 1}$ nor $S_{A 2}$ bands cross the Fermi level. On the other hand, the fully relativistic calculation presents four surface bands labeled as $S_{A 1}^{\downarrow}, S_{A 1}^{\uparrow}, S_{A 2}^{\downarrow}$, and $S_{A 2}^{\uparrow}$. These bands are interpreted as originating from the spin- splitting of the scalar relativistic $S_{A 1}$ and $S_{A 2}$ bands.

It is evident from Fig. 2 that the spin-orbit interaction induces a considerable perturbation on the surface bands associated to the scalar relativistic calculation. The spindegeneracy of the fully relativistic surface bands at the $\bar{\Gamma}$ and $\bar{M}$ points (Fig. 2) is a consequence of the combination of the $\mathrm{C} 3$ rotational and the time reversal symmetry of the system. ${ }^{11}$ In contrast, these symmetry considerations do not forbid a finite spin-orbit energy shift at high symmetry point $\bar{K}$. The insets of Fig. 2 reveal the exact magnitude of the spin-orbit interaction close to $\bar{K}$ point, finding that the $S_{A 1}^{\downarrow}$ and $S_{A 1}^{\uparrow}$ bands are spin-split by $\sim 0.25 \mathrm{eV}$, in good agreement with ARPES photoemission measurements. ${ }^{31}$ In an analogous way, the $S_{A 2}^{\downarrow}$ and $S_{A 2}^{\uparrow}$ bands suffer a maximum splitting of $\sim 0.6 \mathrm{eV}$, an extraordinarily large value for a spin-orbit energy shift. The energy band gap of this termination reduces roughly from a value of $0.2 \mathrm{eV}$ in scalar relativistic calculations to the $0.1 \mathrm{eV}$ found in fully relativistic bands.

In Fig. 3, we present the calculated momentum-dependent spin-polarization for the four spin-splitted states. The spinpolarization is defined as the expectation value of the Pauli matrices

$$
m_{\alpha, i}(\mathbf{k})=\frac{1}{\Omega} \sum_{\sigma \sigma^{\prime}} \int \phi_{\mathbf{k}, i}^{\sigma^{\prime} *}(\mathbf{r}) \sigma_{\alpha}^{\sigma^{\prime} \sigma} \phi_{\mathbf{k}, i}^{\sigma}(\mathbf{r}) d \mathbf{r},
$$

where $\Omega$ denotes the volume of the system and $\alpha$ runs over the cartesian axes. $\phi_{\mathbf{k}, i}^{\sigma}(\mathbf{r})$ represent the Kohn-Sham eigen-spinor of the surface states, while $\sigma_{\alpha}^{\sigma^{\prime} \sigma}$ denote the matrix elements of the Pauli spin-operator. As demonstrated in Fig. 3, a given surface state is spin- polarized in approximately the opposite direction with respect to its associated spin-splitted state. The negligible spin-polarization around high symmetry points $\bar{\Gamma}$

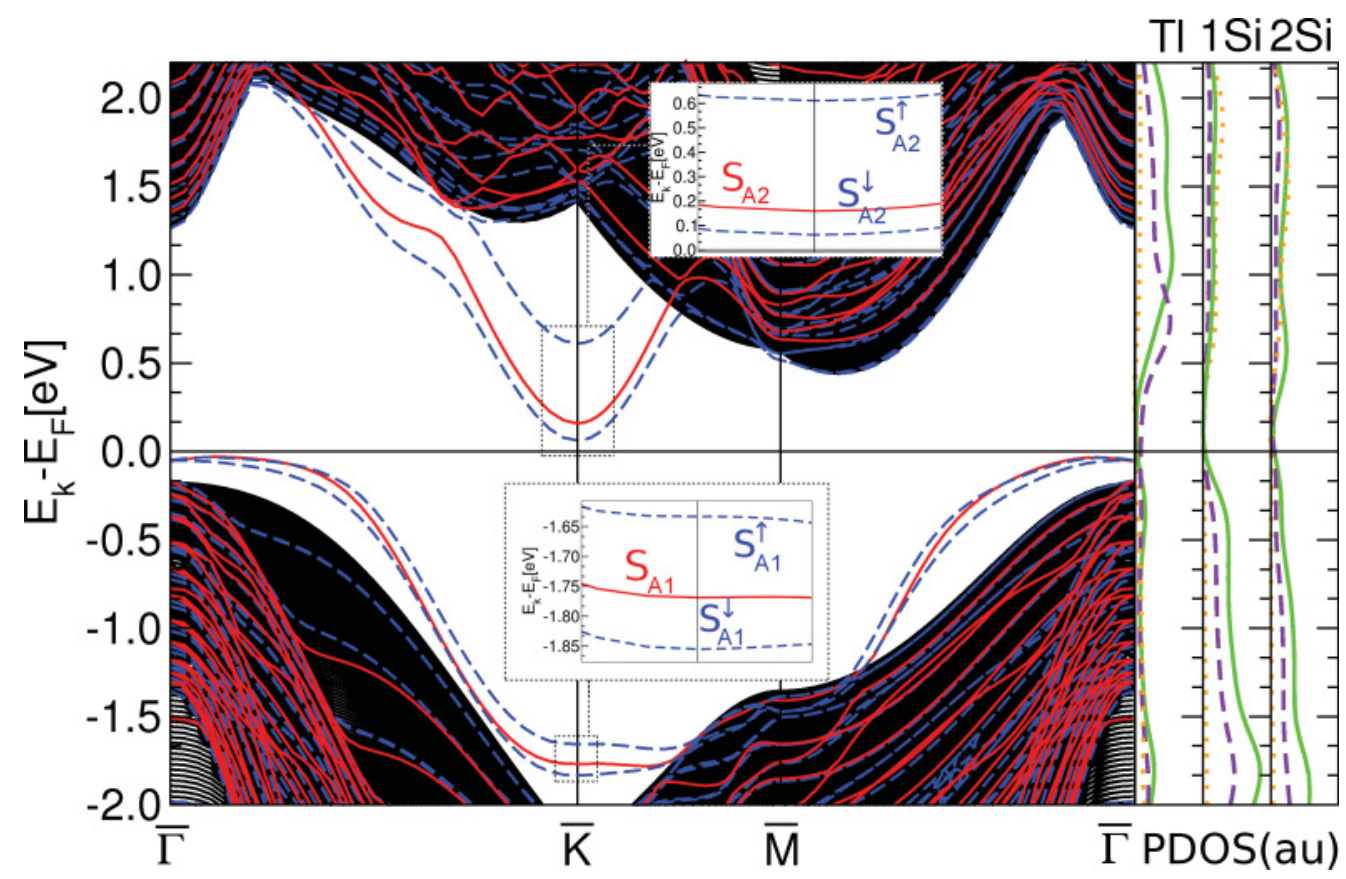

FIG. 2. (Color online) (Left) Band structure of the Tl/Si(111)A surface termination. The scalar relativistic and fully relativistic bands are represented by solid (red) and dashed (blue) lines, respectively. The continuous background denotes the bulk band projection. Insets show the details of the surface bands in the neighborhood of high symmetry point $\bar{K}$. (Right) Projected DOS for the Tl surface monolayer and the first two Si layers. $n p_{3 / 2}, n p_{1 / 2}$, and $n s_{1 / 2}$ orbitals (principal quantum number $n=6$ for $\mathrm{Tl}, n=3$ for $\mathrm{Si}$ ) are represented by solid (green), dashed (violet), and dotted (orange) lines, respectively. 


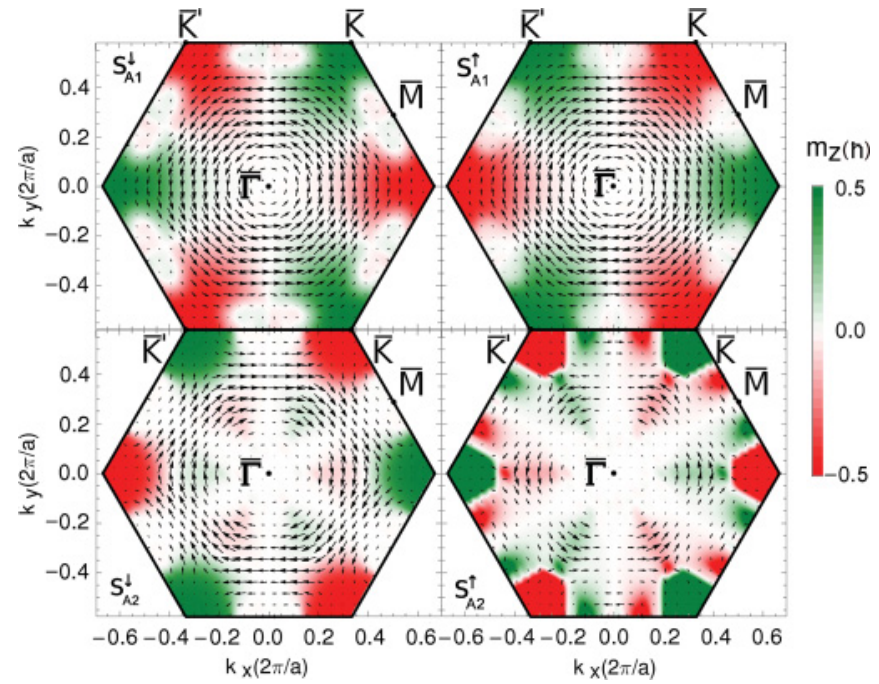

FIG. 3. (Color online) Spin-polarized structure through the entire surface Brillouin zone. Arrows (black) represent the in-plane spinpolarization component, while the background reflects the surface perpendicular component $m_{z, i}(\mathbf{k})$ (the scale ranges $[-0.5 \hbar, 0.5 \hbar]$ ).

and $\bar{M}$ is consistent with the null spin-splitting observed in the band structure in these regions.

It is commonly accepted, on the grounds of the Rashba model, ${ }^{19}$ that the spin-state of the surface electrons is constrained to lie parallel to the surface plane. Figure 3 depicts an in-plane rotational spin-polarization around the $\bar{\Gamma}$ point, qualitatively resembling the Rashba picture. In addition, Fig. 3

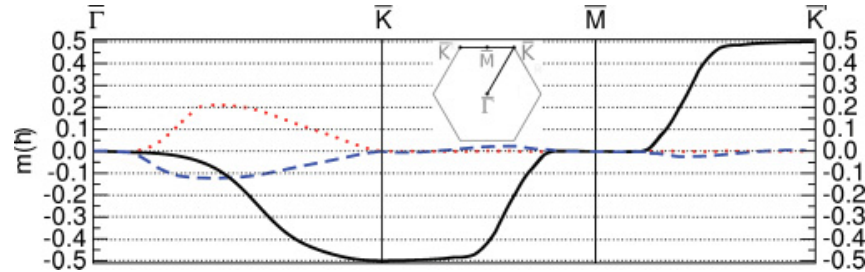

FIG. 4. (Color online) The calculated spin-polarization components $m_{x, S_{A 2}^{\downarrow}}(\mathbf{k}), m_{y, S_{A 2}^{\downarrow}}(\mathbf{k})$, and $m_{z, S_{A 2}^{\downarrow}}(\mathbf{k})$ are represented along the high symmetry line $\bar{\Gamma}-\bar{K}-\bar{M}-\overline{K^{\prime}}$ by dotted (red), dashed (blue), and solid (black) lines, respectively.

reveals that close to the $\bar{K}$ and $\overline{K^{\prime}}$ symmetry points, the spin-state of the surface electrons becomes predominantly polarized along the $z$ direction, in agreement with recent spin-resolved ARPES measurements. ${ }^{31}$ This characteristic property is a consequence of the $\mathrm{C} 3$ rotational symmetry of the honeycomb layered structure of the surface. ${ }^{11}$

In Fig. 4, we present a quantitative analysis of the spinpolarization components of the $S_{A 2}^{\downarrow}$ band along the $\bar{\Gamma}-$ $\bar{K}-\bar{M}-\overline{K^{\prime}}$ high symmetry lines. These results demonstrate that in the neighborhood of high symmetry points $\bar{K}$ and $\overline{K^{\prime}}$, the absolute value of the $z$ component almost reaches the maximum value, $0.5 \hbar$, while the in-plane components become negligible. Furthermore, Fig. 4 indicates that the electronic states around high symmetry points $\bar{K}$ and $\overline{K^{\prime}}$ are spin-polarized in completely opposite directions. These remarkable properties make the $\mathrm{Tl} / \mathrm{Si}(111) \mathrm{A}$ termination a

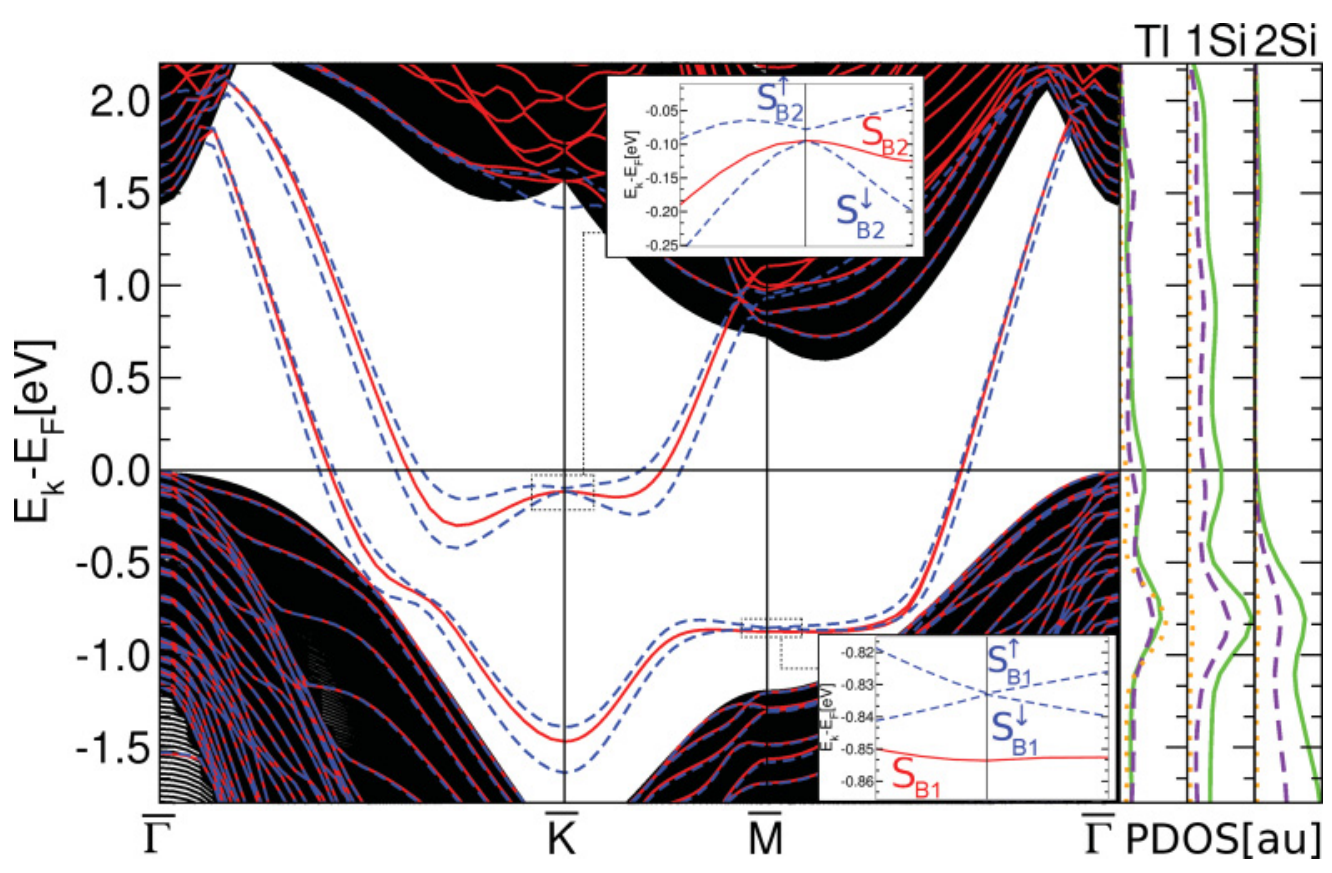

FIG. 5. (Color online) (Left) Band structure of the Tl/Si(111)B surface termination. The scalar relativistic and fully relativistic bands are represented by solid (red) and dashed (blue) lines, respectively. The continuous background denotes the bulk band projection. Inset connected to the $\bar{M}$ point shows a complete spin-degeneracy of $S_{B 1}^{\downarrow}$ and $S_{B 1}^{\uparrow}$ bands. Inset connected to $\bar{K}$ point reveals a quasidegenerate configuration of $S_{B 2}^{\downarrow}$ and $S_{B 2}^{\uparrow}$ bands $\left(\Delta E_{s} \sim 25 \mathrm{meV}\right)$. (Right) Projected DOS for the Tl surface monolayer and the first two Si layers. $n p_{3 / 2}, n p_{1 / 2}$, and $n s_{1 / 2}$ orbitals (principal quantum number $n=6$ for Tl, $n=3$ for Si) are represented by solid (green), dashed (violet), and dotted (orange) lines, respectively. The energy regions around -0.75 and $-0.1 \mathrm{eV}$ show non-negligible $\mathrm{Tl} 6 s$ orbital contribution. 


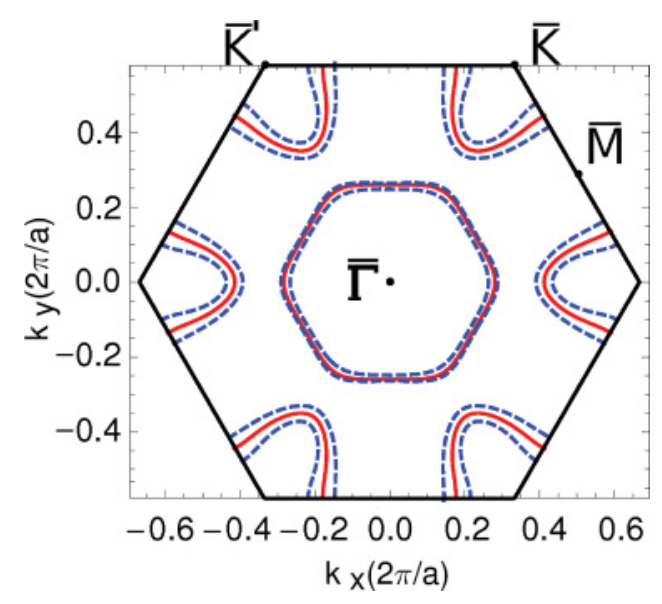

FIG. 6. (Color online) Fully spin-polarized Fermi surface of the $\mathrm{Tl} / \mathrm{Si}$ (111)B surface termination. Solid (red) and dashed (blue) lines represent the Fermi crossing points of scalar relativistic and fully relativistic surface bands, respectively. In the fully relativistic case, the inner and outer electron pockets around $\bar{K}$ and $\overline{K^{\prime}}$ points belong to $S_{B 2}^{\uparrow}$ and $S_{B 2}^{\downarrow}$ bands, while the inner and outer electron-hole pockets around the $\bar{\Gamma}$ point belong to $S_{B 1}^{\downarrow}$ and $S_{B 1}^{\uparrow}$ bands, respectively.

particularly interesting system to study, for instance, the low energy transport properties or optically induced spin-flip transitions. ${ }^{32}$

\section{B. $\mathrm{Tl} / \mathrm{Si}(111) \mathrm{B}$}

The electronic structure of the $\mathrm{Tl} / \mathrm{Si}(111) \mathrm{B}$ termination is presented in Fig. 5. In this termination, we find that four spin-splitted surface bands $\left(S_{B 1}^{\downarrow}, S_{B 1}^{\uparrow}, S_{B 2}^{\downarrow}\right.$, and $\left.S_{B 2}^{\uparrow}\right)$ cross the Fermi level, producing a fully spin-polarized Fermi surface (Fig. 6). The $S_{B 2}^{\downarrow}$ and $S_{B 2}^{\uparrow}$ bands form several spin-polarized electron pockets around the high symmetry points $\bar{K}$ and $\overline{K^{\prime}}$. The $S_{B 1}^{\downarrow}$ and $S_{B 1}^{\uparrow}$ bands are occupied all over the Brillouin zone except around high symmetry point $\bar{\Gamma}$, where we find an electron-hole pocket of radius $k_{F} \sim 0.46 \AA^{-1}$ (see Figs 5 and 6). Consequently, the $\mathrm{Tl} / \mathrm{Si}(111) \mathrm{B}$ termination exhibits a strong metallic character entirely induced by the fully relativistic surface bands.

The $S_{B 1}^{\downarrow}$ and $S_{B 1}^{\uparrow}$ states are maximally spin-split close to the $\bar{K}$ point $(\sim 0.25 \mathrm{eV})$. These bands become spin-degenerate at the $\bar{M}$ point, as it can be appreciated in the inset of Fig. 5. Similarly, the overall spin-splitting for the $S_{B 2}^{\downarrow}$ and $S_{B 2}^{\uparrow}$ surface bands is found to be of the order of $0.2 \mathrm{eV}$. Close to the $\bar{K}$ point, these bands become accidentally quasidegenerate and the magnitude of the splitting diminishes to a negligible but finite value of $\sim 25 \mathrm{meV}$ (inset of Fig. 5).

The $s$ orbital character of the surface electronic wave functions is indicative of a possible spin-degeneracy. The right panel of Fig. 5 shows the projected density of states ${ }^{33}$ (PDOS) for various orbital components. We find that the $\mathrm{Tl}$ $6 s$ orbitals represent the largest contribution to the PDOS at $\sim-0.75 \mathrm{eV}$. Similarly, we find a non-negligible contribution of these orbitals at around $-0.1 \mathrm{eV}$. These two energy regions with non-negligible $\mathrm{Tl} 6 s$ contribution coincide with the energy regions of the insets of Fig. 5.

Figure 7 illustrates several charge isosurfaces $(\rho=$ $\left.\sum_{\sigma}\left|\phi_{\mathbf{k}, i}^{\sigma}(\mathbf{r})\right|^{2}\right)$ associated to the $i=S_{B 2}^{\downarrow}$ state at high symmetry point $\bar{K}$. As demonstrated in the figure, this surface state is localized within the first two layers of the slab. Close to the Tl atoms, where relativistic effects prevail, the charge distribution shows a characteristic "front lobe" shape associated to an atomic $s p_{z}$ hybrid orbital. The big isosurface volume of the "front lobe" indicates that the $s$ character predominates over the $p_{z}$. Interestingly, close to the $\bar{M}$ point, very similar charge distributions are found for the $S_{B 1}^{\downarrow}$ and $S_{B 1}^{\uparrow}$ states, concluding that, effectively, the Tl $6 s$ character is predominant in the spin-degenerate regions.

Figure 8 presents the calculated spin-polarization for the different surface states of the $\mathrm{Tl} / \mathrm{Si}(111) \mathrm{B}$ termination, exhibiting a far more complex structure than in the previous

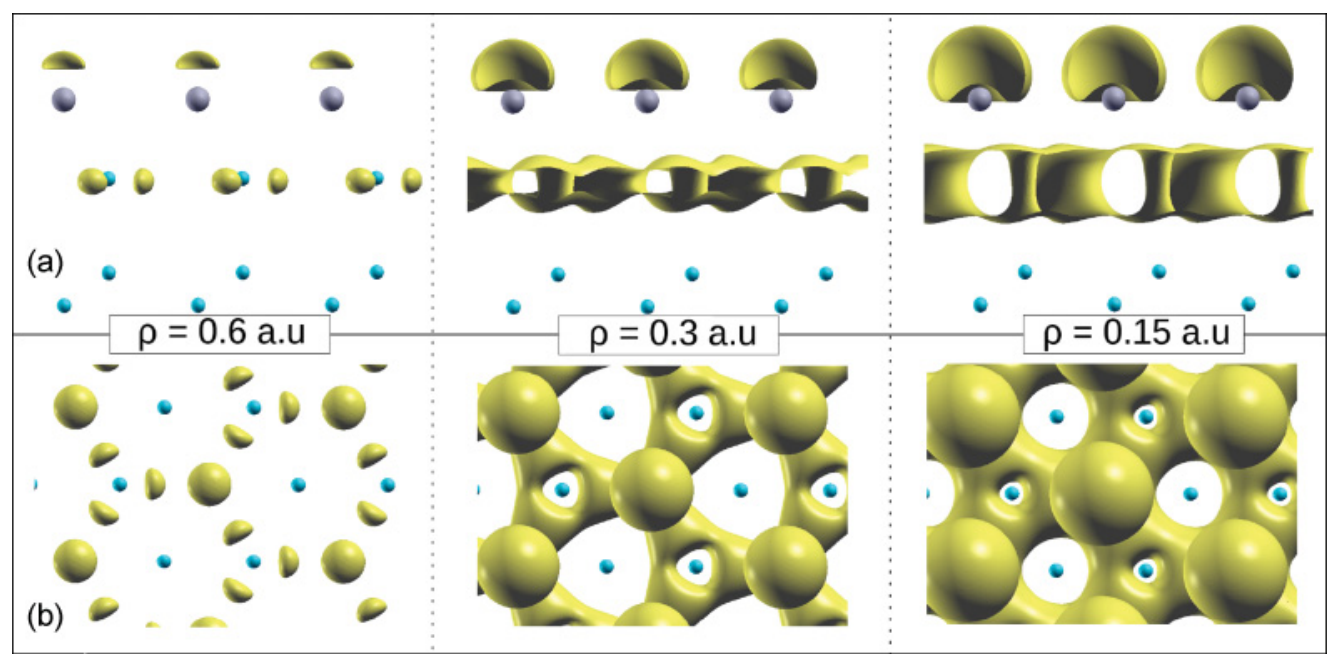

FIG. 7. (Color online) Charge distribution of $S_{B 2}^{\downarrow}$ state at high symmetry point $\bar{K}$. Big (gray) and small (blue) spheres represent Tl and $\mathrm{Si}$ atoms, respectively. (a) and (b) The side and top views of the charge density isosurfaces corresponding to $\rho=0.6,0.3$, and 0.15 a.u. The shape of the charge distribution around $\mathrm{Tl}$ atoms shows a "front lobe" associated to a $s p_{z}$ hybrid orbital with a predominant $s$ to $p_{z}$ ratio. An almost identical picture is obtained for the $S_{B 2}^{\uparrow}$ state. 


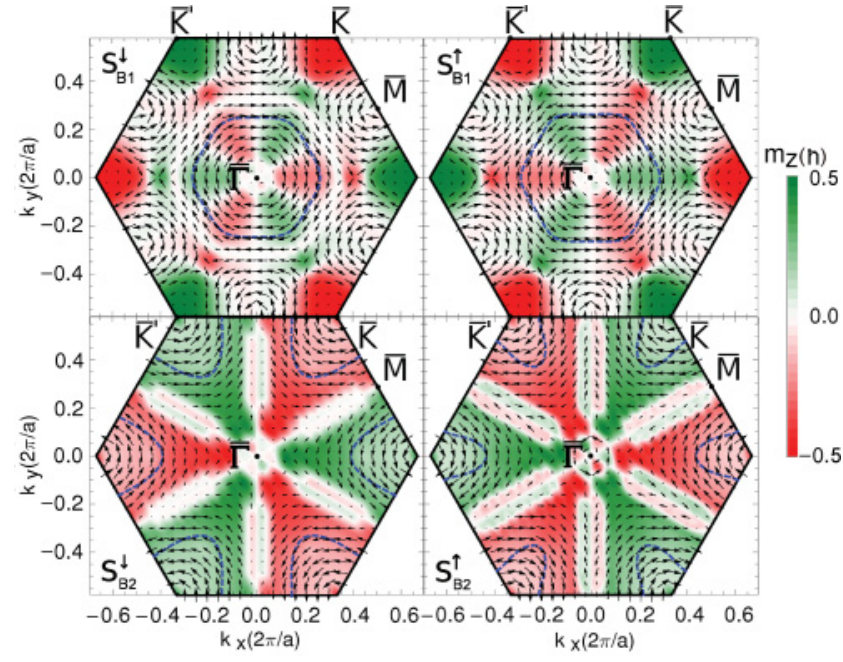

FIG. 8. (Color online) Spin-polarized structure through the entire surface Brillouin zone. Arrows (black) represent the in-plane spinpolarization component, while the background reflects the surface perpendicular component $m_{z, i}(\mathbf{k})$. The Fermi surface for each state is depicted by the dashed (blue) lines.

termination. In agreement with symmetry considerations, the spin is found to be $100 \%$ polarized along the surface perpendicular direction at high symmetry points $\bar{K}$ and $\overline{K^{\prime}}$, its orientation being reversed going from one point to the other.

Our ab initio calculations demonstrate an important contribution of the $z$ spin-polarized component over the entire Brillouin zone, specially for the $S_{B 2}^{\downarrow}$ and $S_{B 2}^{\uparrow}$ states. This behavior strongly departs from simple model calculations predicting a surface perpendicular spin-polarization only in a very small area around $\bar{K}$ and $\overline{K^{\prime}} .{ }^{11}$ The calculated Fermi surface extends over the regions where the in-plane spin-polarization is combined with an important surface perpendicular contribution (see Fig. 8). Interestingly, the spin-polarization reverses its orientation over the different electron pockets, providing the $\mathrm{Tl} / \mathrm{Si}(111) \mathrm{B}$ termination with unique transport properties. A qualitative aspect revealed by these calculations is that the spin is rotational and encircling an appreciable area around all the high symmetry points of the Brillouin zone $(\bar{\Gamma}, \bar{M}$, and $\bar{K}$ ). In conclusion, the spin-polarization structure in Fig. 8 evidences that the details of a surface termination are sufficient to produce highly complex spin-patterns in reciprocal space, beyond simple theoretical models.

\section{CONCLUSIONS}

In summary, we analyze the relativistic electron and spin-structure of two different terminations of the $\mathrm{Tl} / \mathrm{Si}(111)$ surface, $\mathrm{Tl} / \mathrm{Si}(111) \mathrm{A}$ and $\mathrm{Tl} / \mathrm{Si}(111) \mathrm{B}$. The calculations on the A termination are in very good agreement with spin-resolved ARPES photoemission experiments. ${ }^{31}$ Our analysis demonstrates that the band gap of this surface is reduced to a value of $\sim 0.1 \mathrm{eV}$ as a direct consequence of the spin-orbit interaction, the Fermi level being completely surrounded by fully spinpolarized surface states. In overall, both terminations show a highly complex spin-polarization structure in momentum space, particularly the $\mathrm{Tl} / \mathrm{Si}(111) \mathrm{B}$ termination. For this surface, we find that several spin-rotational centers are present, and that the in-plane spin-polarization is combined with a substantial surface perpendicular component over the entire Brillouin zone. All these features strongly depart from simple model theoretical predictions. The $\mathrm{Tl} / \mathrm{Si}(111) \mathrm{B}$ termination possess a strong metallic character entirely induced by the relativistic surface bands and the calculated Fermi surface is mainly constituted by fully spin-polarized electron pockets around high symmetry points $\bar{K}$ and $\overline{K^{\prime}}$. It is found that the spin-polarization reverses its orientation between different electron pockets and thus remarkable transport properties should be expected for both surfaces.

\section{ACKNOWLEDGMENTS}

The authors are grateful to M. Martinez-Canales, B. Rousseau, and I. Errea for fruitful discussions, and acknowledge financial support from UPV/EHU (Grant No. IT-366-07) and the Spanish Ministry of Science and Innovation (Grant No. FIS2010-19609-C02-00). Computer facilities were provided by the Donostia International Physics Center (DIPC).
${ }^{1}$ S. Datta and B. Das, Appl. Phys. Lett. 56 (1990).

${ }^{2}$ F. S. M. Guimaraes, A. T. Costa, R. B. Muniz, and M. S. Ferreira, Phys. Rev. B 81, 233402 (2010).

${ }^{3}$ J. Nitta, T. Akazaki, H. Takayanagi, and T. Enoki, Phys. Rev. Lett. 78, 1335 (1997).

${ }^{4}$ D. D. Awschalom and M. E. Flatte, Nat. Phys. 3, 153 (2007).

${ }^{5}$ S. LaShell, B. A. McDougall, and E. Jensen, Phys. Rev. Lett. 77, 3419 (1996).

${ }^{6}$ A. Eiguren and C. Ambrosch-Draxl, New J. Phys. 11, 013056 (2009).

${ }^{7}$ J. I. Pascual, G. Bihlmayer, Yu. M. Koroteev, H.-P. Rust, G. Ceballos, M. Hansmann, K. Horn, E. V. Chulkov, S. Blügel, P. M. Echenique, and Ph. Hofmann, Phys. Rev. Lett. 93, 196802 (2004).

${ }^{8}$ K. Sugawara, T. Sato, S. Souma, T. Takahashi, M. Arai, and T. Sasaki, Phys. Rev. Lett. 96, 046411 (2006).

${ }^{9}$ J. H. Dil, J. Phys. Condens. Matter 21, 403001 (2009).
${ }^{10}$ A. Eiguren and C. Ambrosch-Draxl, Phys. Rev. Lett. 101, 036402 (2008).

${ }^{11}$ M.-H. Liu and C.-R. Chang, Phys. Rev. B 80, 241304 (2009).

${ }^{12}$ J. Premper, M. Trautmann, J. Henk, and P. Bruno, Phys. Rev. B 76, 073310 (2007).

${ }^{13}$ F. Meier, H. Dil, J. Lobo-Checa, L. Patthey, and J. Osterwalder, Phys. Rev. B 77, 165431 (2008).

${ }^{14}$ K. Yaji, Y. Ohtsubo, S. Hatta, H. Okuyama, K. Miyamoto, T. Okuda, A. Kimura, H. Namatame, M. Taniguchi, and T. Aruga, Nat. Commun. 1, 17 (2010).

${ }^{15}$ C. R. Ast, J. Henk, A. Ernst, L. Moreschini, M. C. Falub, D. Pacilé, P. Bruno, K. Kern, and M. Grioni, Phys. Rev. Lett. 98, 186807 (2007).

${ }^{16}$ K. Sakamoto, K. Haruya, K. Sugawara, K. Miyamoto, A. Kimura, T. Kuzumaki, N. Ueno, E. Annese, J. Fujii, A. Kodama et al., Phys. Rev. Lett. 103, 156801 (2009).

${ }^{17}$ P. S. Nayar and W. O. Hamilton, Appl. Opt. 16, 2942 (1977). 
${ }^{18}$ A. Baumgartner, T. Ihn, K. Ensslin, G. Papp, F. Peeters, K. Maranowski, and A. C. Gossard, Phys. Rev. B 74, 165426 (2006).

${ }^{19}$ E. I. Rashba, Sov. Phys. Solid State 2, 1109 (1960).

${ }^{20}$ P. Giannozzi, S. Baroni, N. Bonini, M. Calandra, R. Car, C. Cavazzoni, D. Ceresoli, G. L. Chiarotti, M. Cococcioni, I. Dabo et al., J. Phys. Condens. Matter 21, 395502 (2009).

${ }^{21}$ A. Dal Corso and A. Mosca Conte, Phys. Rev. B 71, 115106 (2005).

${ }^{22}$ P. E. Blöchl, O. Jepsen, and O. K. Andersen, Phys. Rev. B 49, 16223 (1994).

${ }^{23}$ H. J. Monkhorst and J. D. Pack, Phys. Rev. B 13, 5188 (1976).

${ }^{24}$ J. P. Perdew, K. Burke, and M. Ernzerhof. Phys. Rev. Lett. 77, 3865 (1996).

${ }^{25}$ J. E. Peralta, G. E. Scuseria, and M. J. Frisch, Phys. Rev. B 75, 125119 (2007).
${ }^{26}$ L. Kleinman and D. M. Bylander, Phys. Rev. Lett. 48, 1425 (1982).

${ }^{27}$ G. Theurich and N. A. Hill, Phys. Rev. B 64, 073106 (2001).

${ }^{28}$ L. Kleinman, Phys. Rev. B 21, 2630 (1980).

${ }^{29}$ T. Noda, S. Mizuno, J. Chung, and H. Tochihara. Jpn. J. Appl. Phys. 42, L319 (2003).

${ }^{30}$ G. Lee, C. G. Hwang, N. D. Kim, J. Chung, J. S. Kim, and S. Lee, Phys. Rev. B 76, 245409 (2007).

${ }^{31}$ K. Sakamoto, T. Oda, A. Kimura, K. Miyamoto, M. Tsujikawa, A. Imai, N. Ueno, H. Namatame, M. Taniguchi, P. E. J. Eriksson, and R. I. G. Uhrberg, Phys. Rev. Lett. 102, 096805 (2009).

${ }^{32}$ V. K. Dugaev, E. Ya. Sherman, and J. Barnaś, Phys. Rev. B 83, 085306 (2011).

${ }^{33}$ D. Sanchez-Portal, E. Artacho, and J. M. Soler, Solid State Commun. 95, 685 (1995). 